\title{
What goes around comes around: modeling malaria transmission from humans back to mosquitos
}

\author{
Kazutoyo Miura' and Peter D. Crompton² \\ 'Malaria Immunology Section, Laboratory of Malaria and Vector Research, and 2 Malaria Infection Biology and Immunity Section, Laboratory of Immunogenetics, National Institute of Allergy and Infectious \\ Diseases, NIH, Rockville, Maryland, USA.
}

\begin{abstract}
Malaria, caused by mosquito-transmitted Plasmodium parasites, continues to take a major toll on global health. The development of drugs and vaccines that reduce malaria transmission from humans back to mosquitos could contribute to the control and eventual eradication of malaria, but research models for the early clinical evaluation of candidate interventions are lacking. In this issue of the $J C I$, Collins and colleagues report the successful transmission of Plasmodium falciparum parasites from humans to mosquitoes during controlled human malaria infection, thus providing a potential tool to accelerate the development of much needed transmissionblocking drugs and vaccines.
\end{abstract}

\section{Need for developing new tools for combating malaria}

Malaria is caused by five Plasmodium parasite species, of which Plasmodium falciparum inflicts the greatest disease burden, causing approximately 200 million cases of malaria and 500,000 deaths each year (1). $P$. falciparum infection begins when mosquitos inject sporozoites into the skin and blood. Sporozoites rapidly migrate to the liver, where they replicate asexually before reentering the bloodstream and invading erythrocytes, causing fever and other malaria symptoms. A small percentage of bloodstage parasites differentiate into nonpathogenic, sexual forms called gametocytes that are taken up by the mosquito during a blood meal to complete the life cycle (Figure 1A).

Encouragingly, there has been an unprecedented reduction in malaria cases and deaths since the turn of the century that coincided with the scale-up of malaria control efforts, particularly in sub-Saharan Africa (2). However, the WHO recently reported that progress against malaria may have stalled and even reversed in some regions (1). This sobering news emphasizes the need to not only expand the use of existing tools to fight malaria - namely, antimalarial drugs, mosquito bed nets, and insecticides - but also to develop new tools to further reduce malaria transmission and contribute to its eventual eradication (3). A key component of this strategy is the development of vaccines and drugs that have the ability to reduce or block malaria transmission from humans back to the mosquito vector (4). However, the development of such tools has been impeded, in part, by a lack of practical and reproducible in vivo models for the early evaluation of candidate transmission-blocking interventions. In this issue of the JCI, Katharine Collins and colleagues report the safe and reproducible induction of $P$. falciparum gametocytes during controlled human malaria infection (CHMI) and demonstrate their transmissibility to mosquitoes (5), thereby moving the field toward a physiologically relevant model that could accelerate the evaluation and prioritization of novel transmission-blocking interventions.

\section{The CHMI model}

Since the early 1900s, humans have been deliberately infected with Plasmodium

Related Article: p. 1551

Conflict of interest: The authors have declared that no conflict of interest exists

Reference information: / Clin Invest. 2018;128(4):1264-1266. https://doi.org/10.1172/JCI120260.

parasites for both therapeutic and research purposes. This colorful history began with the intentional infection of neurosyphilis patients with Plasmodium vivax to induce high fevers that were thought to kill treponemal spirochetes in the central nervous system (6). By the 1940s, scientists were infecting humans with Plasmodium to test antimalarial drugs, and by the 1970 s, to investigate the efficacy of malaria immunization (7). In recent years, CHMI has become a common research tool for evaluating candidate drugs and vaccines that target the liver and asexual blood stages (8), and it is now performed at many centers around the world, including sites in malaria-endemic regions of Africa (9, 10). CHMI can be initiated by the bites of Plasmodium-infected mosquitos, by injecting sporozoites dissected from mosquito salivary glands (11), or by sidestepping the liver altogether and inoculating infected erythrocytes intravenously (12). Regardless of how CHMI is initiated, it is terminated with a curative course of antimalarial drugs once asexual parasites are detected in blood. At this stage, mature $P$. falciparum gametocytes are not typically detectable, since their appearance in circulation lags behind that of asexual parasites by approximately 10 days, thus precluding any rigorous study of gametocytes or transmission-blocking interventions with standard CHMI models.

\section{Efficacy of the CHMI model of gametocyte transmission} With the goal of developing a CHMI model of gametocyte transmission, Collins and colleagues modified their CHMI protocol by using piperaquine monotherapy (5), taking advantage of the drug's selective activity against asexual blood-stage parasites (Figure 1B and ref. 13). In this study, $P$. falciparum-infected erythrocytes were administered intravenously to 17 malarianaive adults, and seven to eight days later, a single oral dose of piperaquine cleared 
A

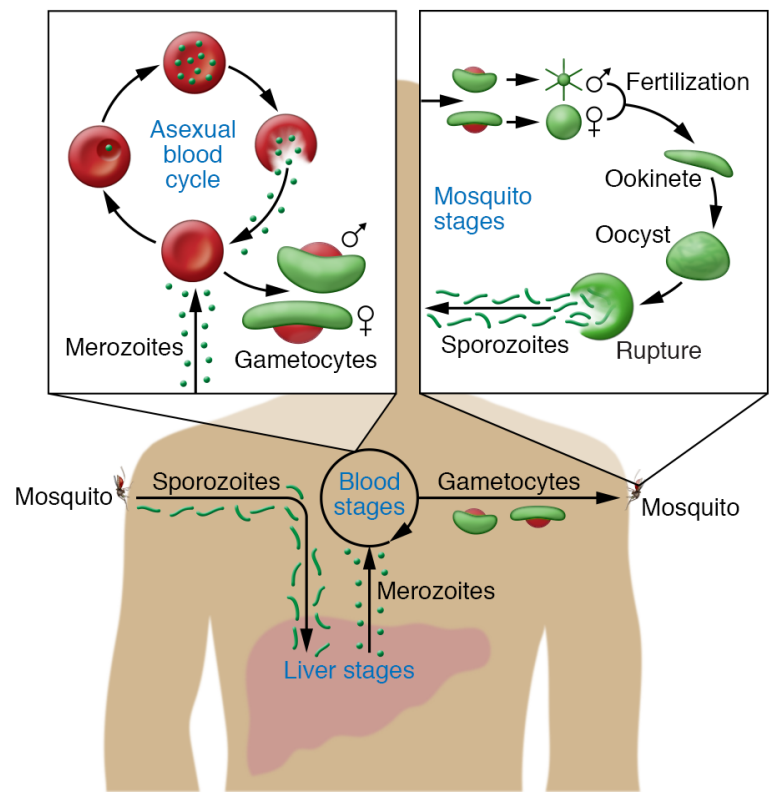

B

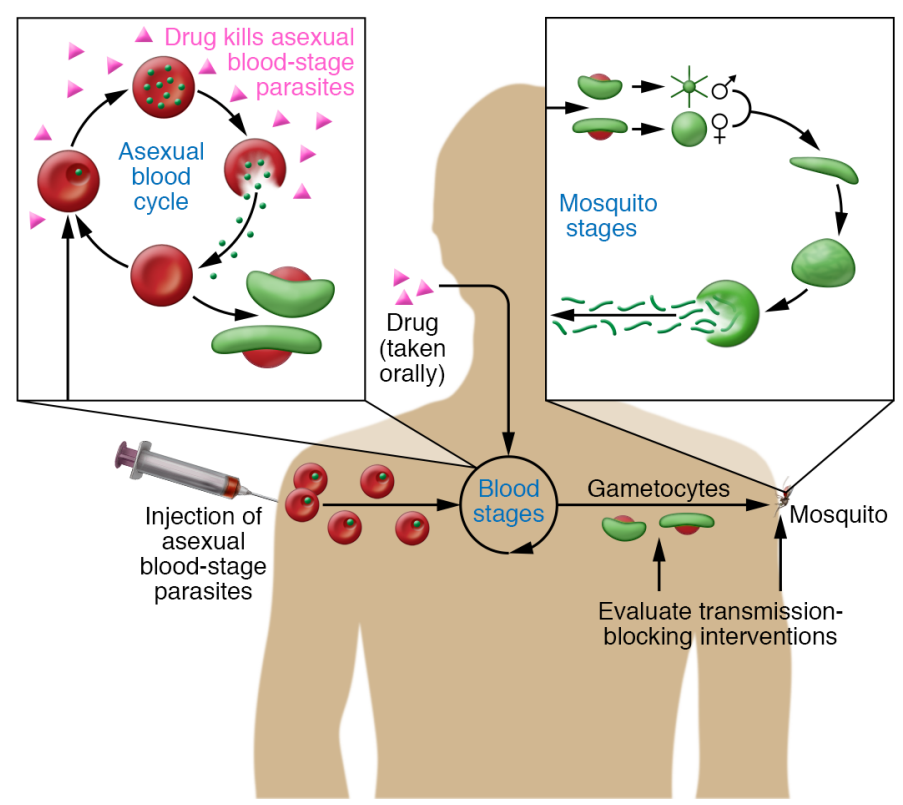

Figure 1. P. falciparum malaria: the life cycle of natural infection and a new model of human-to-mosquito transmission. (A) Natural $P$. falciparum infection begins in humans when Anopheles mosquitos inject sporozoites into the skin and blood. Sporozoites migrate to the liver and invade hepatocytes, where they differentiate into merozoites and replicate asexually without causing symptoms. After seven to ten days, merozoites exit the liver into the bloodstream and begin a 48-hour cycle of asexual replication within erythrocytes. Asexual blood-stage parasites cause disease by triggering inflammation and sequestering in blood vessels of vital organs. A small percentage of blood-stage parasites differentiate into male and female gametocytes - the nonpathogenic sexual forms taken up by mosquitos during blood meals. In the mosquito, gametes fuse and ultimately form sporozoites that migrate to the mosquito salivary gland to complete the life cycle. (B) In this issue, Collins and colleagues describe a new model of malaria transmission from humans to mosquitoes using CHMI. P. falciparum-infected erythrocytes were inoculated intravenously into malaria-naive adults, bypassing the liver and directly establishing a blood-stage infection. The drug piperaquine, which is selectively active against asexual blood-stage parasites, was administered orally seven to eight days later to attenuate the replication of pathogenic asexual parasites while allowing nonpathogenic gametocytes to develop and mature. The transmissibility of gametocytes from humans to mosquitos was assessed either directly by feeding laboratory-reared mosquitoes on the skin of infected subjects or indirectly with assays in which infected blood is fed to mosquitoes through an artificial membrane. All subjects received a curative course of antimalarial drugs at the end of the study.

asexual parasites from five subjects, while the remaining subjects required a second higher dose of piperaquine. As early as 10 days after inoculation, male and female gametocytes began appearing in the circulation of all subjects and remained at stable levels for up to 21 days, when a gametocytocidal drug was administered to end the study. At several time points between the appearance of gametocytes and the end of the study, 11 subjects were evaluated for transmissibility, eight of which successfully infected mosquitoes, either by direct skin feeding or with membrane-feeding assays in which gametocyte-infected blood was fed to mosquitoes through an artificial membrane. However, the efficiency of mosquito infection was low, with a median of $7 \%$ of mosquitos becoming infected after direct skin feeding and even fewer after membrane feeding. This low transmission efficiency was not entirely unexpected, as comparable mosquito infection rates have been observed from naturally infected individuals in endemic areas with similar levels of gametocytemia (14).

The authors explored the model's ability to test gametocytocidal drug activity in vivo. Primaquine, a known gametocytocidal drug, reduced gametocytemia by $90 \%$, compared with a $21 \%$ reduction with the investigational antimalarial artefenomel (15), similar to what was seen in those who received no drug. This highlights the model's potential to fill a critical gap in the gametocytocidal drug pipeline - to assess in vivo the safety, dosing, and efficacy of candidate compounds identified through in vitro screens of chemical libraries (16). Although a positive association between gametocytemia and mosquito infection was observed in the model, the relationship between these parameters is influenced by many factors, including the stage of gametocyte maturation and ratio of male and female gametocytes. Without an estab- lished biomarker that predicts transmissibility of gametocytes to mosquitoes (17), direct skin-feeding or membrane-feeding experiments are necessary to accurately estimate transmission-blocking activity, unless an intervention completely clears gametocytes from circulation. However, as the authors note, mosquito infection rates were too low in their model to evaluate the transmission-blocking activity of the drugs tested. By concentrating gametocytes in the membrane-feeding assay, higher infection rates were achieved, and in a pilot experiment, transmission occurred in two of two subjects before primaquine treatment and zero of two subjects after treatment, indicating that higher gametocytemia would improve the model's ability to accurately estimate transmission-blocking activity of candidate drugs and vaccines. It remains unclear how high gametocyte levels must be to feasibly evaluate transmission-blocking interventions with CHMI. Field studies 
in endemic areas show that individuals carrying more than 100,000 gametocytes/ $\mathrm{ml}$ of blood transmit to approximately $25 \%$ of mosquitos through direct and membrane-feeding assays (14). Assuming similar assay performance in field and CHMI studies, the peak gametocytemia attained in the CHMI model (geometric mean of approximately 1,000 gametocytes $/ \mathrm{ml}$ ) would need to be approximately 100fold higher to achieve mosquito infection rates of approximately $25 \%$. The authors speculate that iterative testing of different parasite lines and mosquito vectors might increase gametocytemia and infection rates, thereby achieving sufficient statistical power to detect transmission-blocking activity with a feasible number of subjects. Further efforts to optimize the model are clearly worthwhile, as it would allow candidate transmission-blocking interventions to be prioritized for subsequent studies of naturally infected individuals in endemic areas, which, while highly relevant, are expensive, logistically challenging, and complicated by many factors, including variability in gametocytemia over time and between individuals (15), microheterogeneity of malaria exposure in populations (18), mosquito and human immune factors $(19,20)$, and the presence of antimalarial drugs that affect transmission (21).

\section{Future directions}

As with any model, there is a delicate balance between controlling variables and attempting to stay true to the "real world." In this vein, there are many questions to address going forward. For example, does the gametocyte biology of the laboratoryadapted $P$. falciparum line used in this model differ from that of parasites circulating in nature? Although piperaquine is not frankly gametocytocidal, does it affect gametocyte biology and transmissibility? In a rodent malaria model, mosquito transmission intrinsically modifies the biology of asexual blood-stage parasites (22) - how does initiating CHMI by direct intravenous inoculation (bypassing the mosquito and liver) affect gametocyte biology and transmissi- bility? It will also be of interest to further investigate in vivo differences between male and female gametocytes, such as their relative kinetics and susceptibility to transmission-blocking interventions (23). Despite its current limitations and important unanswered questions, this innovative model promises to continue to deepen our understanding of gametocyte biology and transmission, thereby accelerating the development of novel and urgently needed transmission-blocking interventions.

\section{Acknowledgments}

This work was supported by the Intramural Research Program of the National Institute of Allergy and Infectious Diseases, NIH.

Address correspondence to: Peter D. Crompton, 5625 Fishers Lane, Room 4N07D, Rockville, Maryland 20852, USA. Phone:301.761.5042; Email:pcrompton@ niaid.nih.gov.

1. World Health Organization. World Malaria Report 2017. WHO Website. http://www.who. int/malaria/publications/world-malariareport-2017/en/. Accessed February 20, 2018.

2. Bhatt $\mathrm{S}$, et al. The effect of malaria control on Plasmodium falciparum in Africa between 2000 and 2015. Nature. 2015;526(7572):207-211.

3. Rabinovich RN, et al. malERA: An updated research agenda for malaria elimination and eradication. PLoS Med. 2017;14(11):e1002456.

4. malERA Refresh Consultative Panel on Tools for Malaria Elimination. malERA: An updated research agenda for diagnostics, drugs, vaccines, and vector control in malaria elimination and eradication. PLoS Med. 2017;14(11):e1002455.

5. Collins KA, et al. A controlled human malaria infection model enabling evaluation of transmission-blocking interventions. J Clin Invest. 2018;128(4):1551-1562.

6. Austin SC, Stolley PD, Lasky T. The history of malariotherapy for neurosyphilis. Modern parallels. JAMA. 1992;268(4):516-519.

7. Clyde DF, Most H, McCarthy VC, Vanderberg JP. Immunization of man against sporoziteinduced falciparum malaria. Am JMed Sci. 1973;266(3):169-177.

8. Stanisic DI, McCarthy JS, Good MF. Controlled human malaria infection: applications, advances, and challenges. Infect Immun. 2018;86(1):e00479-17.

9. Hodgson SH, et al. Evaluating controlled human malaria infection in Kenyan adults with varying degrees of prior exposure to Plasmodium falci- parum using sporozoites administered by intramuscular injection. Front Microbiol. 2014;5:686.

10. Shekalaghe S, et al. Controlled human malaria infection of Tanzanians by intradermal injection of aseptic, purified, cryopreserved Plasmodium falciparum sporozoites. Am J Trop Med Hyg. 2014;91(3):471-480.

11. Roestenberg M, et al. Controlled human malaria infections by intradermal injection of cryopreserved Plasmodium falciparum sporozoites. Am J Trop Med Hyg. 2013;88(1):5-13.

12. Engwerda CR, Minigo G, Amante FH, McCarthy JS. Experimentally induced blood stage malaria infection as a tool for clinical research. Trends Parasitol. 2012;28(11):515-521.

13. Pasay CJ, et al. Piperaquine monotherapy of drug-susceptible Plasmodium falciparum infection results in rapid clearance of parasitemia but is followed by the appearance of gametocytemia. J Infect Dis. 2016;214(1):105-113.

14. Bousema T, et al. Mosquito feeding assays to determine the infectiousness of naturally infected Plasmodium falciparum gametocyte carriers. PLoS One. 2012;7(8):e42821.

15. Phyo AP, et al. Antimalarial activity of artefenomel (OZ439), a novel synthetic antimalarial endoperoxide, in patients with Plasmodium falciparum and Plasmodium vivax malaria: an open-label phase 2 trial. Lancet Infect Dis. 2016;16(1):61-69.

16. Birkholtz LM, Coetzer TL, Mancama D, Leroy D, Alano P. Discovering new transmission-blocking antimalarial compounds: challenges and opportunities. Trends Parasitol. 2016;32(9):669-681.

17. malERA Refresh Consultative Panel on Basic Science Enabling Technologies. malERA: An updated research agenda for basic science and enabling technologies in malaria elimination and eradication. PLOS Med. 2017;14(11):e1002451.

18. Mogeni P, et al. Effect of transmission intensity on hotspots and micro-epidemiology of malaria in sub-Saharan Africa. BMC Med. 2017;15(1):121.

19. Bousema T, et al. Human immune responses that reduce the transmission of Plasmodium falciparum in African populations. Int J Parasitol. 2011;41(3-4):293-300.

20. Crompton PD, et al. Malaria immunity in man and mosquito: insights into unsolved mysteries of a deadly infectious disease. Annu Rev Immunol. 2014;32:157-187.

21. Kone A, et al. Sulfadoxine-pyrimethamine impairs Plasmodium falciparum gametocyte infectivity and Anopheles mosquito survival. Int J Parasitol. 2010;40(10):1221-1228.

22. Spence PJ, et al. Vector transmission regulates immune control of Plasmodium virulence. Nature. 2013;498(7453):228-231.

23. White NJ, et al. Assessment of therapeutic responses to gametocytocidal drugs in Plasmodium falciparum malaria. Malar J. 2014;13:483. 